\title{
The dosimetric effects of limited elective nodal irradiation in volumetric modulated arc therapy treatment planning for locally advanced non-small cell lung cancer
}

\author{
Mark C. Kenamond ${ }^{1}$, R. Alfredo Siochi ${ }^{2}$, and Malcolm D. Mattes ${ }^{2}$ \\ ${ }^{1}$ West Virginia University School of Medicine, Morgantown, WV, USA \\ 2Department of Radiation Oncology, West Virginia University, One Medical Center Drive, PO Box \\ 9234, Morgantown, WV, USA
}

\begin{abstract}
Objective-Contemporary radiotherapy guidelines for locally advanced non-small cell lung carcinoma (LA-NSCLC) recommend omitting elective nodal irradiation, despite the fact that evidence supporting this came primarily from older reports assessing comprehensive nodal coverage using 3D conformal techniques. Herein, we evaluated the dosimetric implications of the addition of limited elective nodal irradiation (LENI) to standard involved field irradiation (IFI) using volumetric modulated arc therapy (VMAT) planning.
\end{abstract}

Method-Target volumes and organs-at-risk (OARs) were delineated on CT simulation images of 20 patients with LA-NSCLC. Two VMAT plans (IFI and LENI) were generated for each patient. Involved sites were treated to $60 \mathrm{~Gy}$ in 30 fractions for both IFI and LENI plans. Adjacent uninvolved nodal regions, considered high risk based on the primary tumor site and extent of nodal involvement, were treated to $51 \mathrm{~Gy}$ in 30 fractions in LENI plans using a simultaneous integrated boost approach.

\begin{abstract}
Results-All planning objectives for PTVs and OARs were achieved for both IFI and LENI plans. LENI resulted in significantly higher esophagus $D_{\text {mean }}(15.3$ vs. $22.5 \mathrm{~Gy}, p<0.01$ ), spinal cord $D_{\max }(34.9$ vs. $42.4 \mathrm{~Gy}, p=0.02)$ and lung $D_{\text {mean }}(13.5$ vs. $15.9 \mathrm{~Gy}, p=0.02), V_{20}(23.0$ vs. $27.9 \%, p=0.03$ ), and $V_{5}(52.6$ vs. $59.4 \%, p=0.02)$. No differences were observed in heart parameters. On average, only $32.2 \%$ of the high-risk nodal volume received an incidental dose of 51 Gy when untargeted in IFI plans.

Conclusion-The addition of LENI to VMAT plans for LA-NSCLC is feasible, with only modestly increased doses to OARs and marginal expected increase in associated toxicity.
\end{abstract}

Correspondence to: Malcolm D. Mattes.

Electronic supplementary material The online version of this article (https://doi.org/10.1007/s13566-017-0327-2) contains supplementary material, which is available to authorized users.

Compliance with ethical standards

Conflict of interest The authors declare that they have no conflict of interest.

Ethical approval This article does not contain any studies with human participants or animals performed by any of the authors. Informed consent Statement of informed consent was not applicable since the manuscript does not contain any patient data. 


\section{Keywords}

Lung cancer; Lymph node; Radiation therapy; Non-small cell lung cancer; Intensity modulated radiotherapy

\section{Introduction}

Current guidelines recommend omission of elective nodal irradiation (ENI) in favor of involved field irradiation (IFI) in the definitive management of locally advanced non-small cell lung cancer (LA-NSCLC), with the rationale for this being that smaller target volumes enable lower rates of toxicity due to greater sparing of organs-at-risk (OARs), as well as improved local control by enabling dose escalation to gross disease [1,2]. Omission of ENI is supported by several retrospective series showing only modest increases in nodal recurrence (5-10\%) [3-7], as well as two randomized trials showing improvements in overall survival with IFI $[8,9]$.

However, there are several important caveats when interpreting this data. First, although highly conformal intensity modulated radiation therapy (IMRT) is most commonly used in contemporary practice, the vast majority of studies assessing ENI were carried out using three-dimensional conformal radiation therapy (3DCRT), which delivers a significant "incidental" dose to non-targeted nodal regions that may contribute towards the reportedly low elective nodal recurrence rate $[5,10,11]$. Delivering a similar dose to uninvolved nodal regions with IMRT would require explicit targeting. Furthermore, the reported rates of isolated regional failure may also be artificially low because of ascertainment bias, and even if one does assume a 5-10\% regional recurrence rate, this is relatively substantial considering that only $15-25 \%$ of locally advanced NSCLC will be cured. Finally, the elective nodal volume that was historically treated (including in the above randomized trials) encompassed the ipsilateral hilum, entire mediastinum, and supraclavicular nodal regions, an immense area that would certainly cause undue cardiopulmonary toxicity. In summary, while data exists on all or nothing approaches to ENI using 3DCRT, there is little data on treating more limited elective nodal target volumes using IMRT, and it is not surprising that a recent survey showed great discrepancy in practice patterns for the use of various extents of prophylactic nodal irradiation [12].

The primary goal of this study is to determine the dosimetric impact of limited elective nodal irradiation (LENI) targeting prophylactic regions of the mediastinum at highest risk for harboring microscopic disease using modern treatment planning techniques. We hypothesize that the addition of these limited volumes treated to a lower dose will increase the dose to OARs but to a small enough degree such that all dosimetric objectives can still successfully be met and the expected increase in clinical toxicity is marginal.

\section{Methods}

This is a treatment planning system (TPS)-only study using CT simulation images from a population of 20 patients who were previously treated with definitive radiation therapy for locally advanced NSCLC at our institution from 2015 to 2016. Specific patients were 
selected for inclusion such that the entire cohort would represent a variety of types of target volumes. For instance, we included 10 patients with hilar lymph node involvement only (N1) and 10 patients with mediastinal lymph node involvement (N2), and an equal number of patients with primary tumors located in each of the five lobes of the lung. All patients underwent positron emission tomography/computed tomography (PET/CT) prior to treatment for the purpose of staging and radiotherapy treatment planning, and lymph nodes were considered involved with malignancy if their short axis diameter was greater than $1 \mathrm{~cm}$ on CT or standardized uptake value (SUV) was greater than 3.0 on PET.

All treatment planning was carried out for the Varian TrueBeam linear accelerator (Varian Medical Systems, Palo Alto, CA) using the Eclipse treatment planning system (version 11). All involved field target volumes were contoured according to standard methods described in recent Radiation Therapy Oncology Group (RTOG) clinical trials [13]. Briefly, the gross tumor volume (GTV) consisted of all known gross disease based on the planning CT scan and pretreatment PET/CT scan. An iGTV included the union of the GTVs on all respiratory correlated images on a 4DCT scan acquired at the time of simulation. The clinical target volume (CTV) included the iGTV plus an 8-mm margin to account for miscroscopic extension without extending into uninvolved organs. No uninvolved (elective) nodal regions were added to the CTV for the IFI plans. The CTV was expanded isotropically by $5 \mathrm{~mm}$ to generate a planning target volume that was prescribed a dose of $60 \mathrm{~Gy}$ in 30 fractions (PTV60).

LENI plans utilized the same PTV60 taken to the same prescription dose as the IFI plans; however, an additional planning target volume encompassing adjacent nodal regions at high risk for harboring microscopic disease was also prescribed a dose of $51 \mathrm{~Gy}$ in 30 fractions (PTV51). The LENI plans delivered a dose to the two target volumes in a single plan via a simultaneous integrated boost. The high-risk nodal regions encompassed in the PTV51 were individualized for each patient, based on the patient's primary tumor site and involved nodal stations. Table 1 shows the nodal stations considered to be at high risk in various scenarios, based on known patterns of nodal spread as described in previous surgical series, and guidelines from the LungART clinical trial for postoperative NSCLC patients in which elective nodal coverage is recommended [14-19]. In order to ensure accuracy of the elective nodal contouring, all lymph node stations were contoured as separate structures for every patient using the 2015 International Association for the Study of Lung Cancer (IASLC) lymph node map as a guideline [20]. The elective nodal CTV was generated by combining the contours of the nodal regions considered high risk for a given patient. A 5-mm isotropic expansion was added to generate the PTV51. Finally, OARs were contoured according to the current RTOG consensus atlas [21]. All contouring was carried out under the supervision of a board-certified radiation oncologist.

IFI and LENI plans were generated using volumetric modulated arc therapy (VMAT) using Varian Eclipse RapidArc and the Acuros 10 algorithm for dose calculations. Each plan used $6 \mathrm{MV}$ photos, and either a single or double arc. Equivalent dose-volume constraints for PTV's and OAR's were used for each patient and each plan; however optimization conditions varied on an individual basis depending on the level of difficulty in meeting these constraints for each plan. Each plan was normalized such that 95\% of the PTV60 received 
$100 \%$ of the prescription dose, with an acceptable maximum dose to PTV60 less than $110 \%$ of the prescription dose and an acceptable minimum dose to the PTV60 greater than $90 \%$ of the prescription dose. The OAR objectives can be found in Table 2. Figure 1 shows representative IFI and LENI plans.

Dose-volumetric parameters evaluated for the purpose of comparing the plans included the total lung $V_{20}, V_{5}$, and $D_{\text {mean }}$; heart $V_{60}, V_{45}, V_{30}$, and $D_{\text {mean }}$; esophagus $D_{\max }$ and $D_{\text {mean }}$; and spinal cord $D_{\max }$. $V_{\mathrm{x}}$ is defined as the volume of the OAR receiving at least dose $X(\mathrm{~Gy})$. The maximum and minimum doses were defined as the highest and lowest dose, respectively, within $0.03 \mathrm{~cm}^{3}$ of a given structure. IFI and LENI plans were compared using two-tailed Student's $t$ tests, for both the entire cohort and subsets of patients stratified according to their nodal stage (N1 vs. N2). Finally, for IFI plans, the percentage of each high-risk elective nodal station receiving $51 \mathrm{~Gy}$ was determined for each patient in order to determine the extent of incidental coverage. This study was approved by the local institutional review board.

\section{Results}

The mean (and standard deviation (STD)) dose-volumetric results for all OARs are shown in Table 2. Compared to IFI plans, LENI plans had higher doses to all OARs. However, the average LENI plans still achieved all OAR constraints. LENI resulted in significantly higher esophagus $D_{\text {mean }}\left(15.3\right.$ vs. $22.5 \mathrm{~Gy}, p<0.01$ ), spinal cord $D_{\max }(34.9$ vs. $42.4 \mathrm{~Gy}, p=0.02$ ) and lung $D_{\text {mean }}(13.5$ vs. $15.9 \mathrm{~Gy}, p=0.02), V_{20}(23.0$ vs. $27.9 \%, p=0.03)$, and $V_{5}(52.6$ vs. $59.4 \%, p=0.02)$. No statistically significant differences were observed in heart parameters between IFI and LENI plans. A subgroup analysis of the impact of LENI for stage N1 and N2 patients is shown in Supplementary Table 1. Several statistically significant differences were observed, though the absolute differences between IFI and LENI plans were comparable for N1 and N2 subgroups for each parameter.

Table 3 indicates the percentage of nodal volume outside of the PTV60 receiving at least 51 Gy for IFI plans. On average, only $32.2 \%$ of the high-risk nodal volume targeted in LENI plans received an incidental dose of $51 \mathrm{~Gy}$ when untargeted in IFI plans. Furthermore, on average, only $44.9 \%$ of the uninvolved portions of involved nodal stations received an incidental dose of 51 Gy when untargeted in IFI plans.

\section{Discussion}

Comprehensive ENI using a 3DCRT technique has been previously shown to result in unfavorable outcomes compared to IFI in terms of both toxicity and tumor control. However, to extrapolate this data to contemporary treatment, planning techniques incorporating a more restricted and better selected elective nodal volume is likely to falsely exaggerate the disadvantages of ENI. In this treatment planning system study, we have shown that with the use of VMAT, the addition of LENI treated to a prophylactic dose does not impair the achievement of dosimetric objectives compared to standard IFI for NSCLC. Furthermore, contrary to prior studies assessing 3DCRT plans, we have shown that incidental dose deposition in these high-risk areas is minimal when not explicitly targeted using a VMAT 
plan, and likely inadequate to sterilize microscopic disease. The implications of these findings are not limited to definitive treatment of LA-NSCLC, but are also relevant to small cell lung cancer and post-operative radiation for NSCLC, where some degree of elective nodal coverage is currently more accepted.

Not unexpectedly, the larger target volume in LENI plans was associated with higher dose to OARs despite all dosimetric objectives being achieved. An important question is how these observed dosimetric changes may correlate with actual risks of toxicity in patients.

According to Quantitative Analyses of Normal Tissue Effects in the Clinic (QUANTEC) and Normal Tissue Complications Probability (NTCP) models, the increase in the average mean lung dose from 13.5 to 15.9 Gy with LENI in this study predicts an increased risk of symptomatic pneumonitis of 3.1\% [22]. Similarly, based on the observed increase in the average lung $V_{20}$ from 23.0 to $27.9 \%$, the risk of grade 2 or higher pneumonitis would be increased only 2-3\% [23]. There is limited data available to quantify the risk of pneumonitis at different levels of the lung $V_{5}$, though two studies suggest that an increase from 52.6 to $59.4 \%$ should minimally increase the risk $[24,25]$. The 7.2 -Gy average increase in esophageal $D_{\text {mean }}$ could increase acute esophagitis during treatment to some extent, but because the observed doses were all substantially lower than QUANTEC constraints, and the $D_{\max }$ was below $60 \mathrm{~Gy}$, severe esophageal toxicity would not be expected [22, 26, 27]. The spinal cord $D_{\max }$ did not exceed $50 \mathrm{~Gy}$ in any plan, so no toxicity would be expected during or after this course of treatment [22]. Most nodes were distant from the heart, so heart parameters were minimally affected. For all of these reasons, we expect that the clinical significance of these increased doses to OARs in LENI plans is likely to be marginal.

This is not to say, though, that LENI should be used indiscriminately. For instance, in cases of larger, more central primary tumors with multiple involved mediastinal nodes, the addition of LENI may compromise esophageal constraints. Likewise, if the primary tumor and involved nodal areas encompass a larger longitudinal distance within the lung, implementing LENI may jeopardize the lungs. In our assessment, a risk-adapted approach to treatment planning is appropriate in which the elective target volumes are tailored to a patient's individual situation, in terms of cardio-pulmonary function, age, performance status, and extent of disease.

The expected benefit of LENI based on this study is harder to quantify. Although regional recurrence is not nearly as common as distant recurrence in NSCLC, given the low cure rate of LA-NSCLC, LENI may better optimize the chances of cure for those patients without micrometastatic disease at diagnosis who have a reasonable chance at cure in the first place. What is clear from our data is that the concept of incidental dose going to these other elective nodal areas by only targeting involved areas is simply not true when advanced treatment planning techniques like VMAT with its much greater conformity and more rapid dose falloff (compared to 3DCRT) are used. Specifically, our data demonstrates that the percentage of nodal volume receiving $51 \mathrm{~Gy}$ increases from 32.2 to $99.1 \%$ with the incorporation of LENI. Due to the coplanar nature of most radiotherapy fields, most of this incidental coverage was also in the same axial plane as the gross disease, whereas minimal dose was deposited only a few millimeters superior to it. During surgical excision of NSCLC, a complete resection requires removal of at least one nodal station above the 
highest involved station [28], yet current IFI protocols would fail to deliver dose to this same area unless it were explicitly targeted.

The most important limitation of this study is that it was conducted solely with a treatment planning software and not in actual patients. As such, we could only estimate the effects of the observed dosimetric changes, and assessing the clinical benefit of LENI was beyond our scope. While the number of patients we included would be small for a clinical trial, it is an adequate sample size for a treatment planning system study such as this, and a variety of primary tumor locations and involved nodal stations were chosen intentionally in an attempt to ensure that our findings were generalizable. Another potential limitation is the applicability of our findings to clinicians using different dose levels or other types of IMRT besides VMAT. The 60-Gy dose we used for involved sites is relatively standard since the publication of RTOG 0617 [13], and the 51-Gy dose to elective nodal stations is within the realm of what would be considered adequate to eradicate microscopic disease for most malignancies. Our integrated boost approach to delivering the dose was chosen for simplicity and for a fairer comparison of one IFI plan to one LENI plan (instead of two plans for LENI had we not used an integrated boost). Employing the same radiation technique, dose/fractionation, and normalization were also necessary for a fair comparison between planning techniques. Although the absolute differences may change slightly were we to treat to a higher total dose or use other forms of IMRT planning, we believe that the basic findings highlighted in this study would remain largely intact.

\section{Conclusion}

Herein, we have demonstrated the feasibility of the addition of LENI to standard IFI when using VMAT to treat patients with locally advanced NSCLC, observing only modestly increased doses to OARs and marginal expected increase in associated toxicity. As such, we believe that it should be considered for selected patients likely to benefit in clinical practice.

\section{Supplementary Material}

Refer to Web version on PubMed Central for supplementary material.

\section{Acknowledgments}

We thank Brenda Darnell, Brenda Maxwell, and Robert Bice for teaching and assisting in dosimetric planning and calculations, as well as the West Virginia University Initiation to Research Opportunities (INTRO) Summer Research Program for their support.

Funding No funding was received for this study.

\section{References}

1. National Comprehensive Cancer Network ${ }^{\circledR}$ (NCCN). Practice guidelines in oncology: non-small cell lung cancer, Version 4. 2017. www.nccn.org

2. Belderbos JS, Kepka L, Spring Kong FM, Martel MK, Videtic GM, Jeremic B. Report from the International Atomic Energy Agency (IAEA) consultants' meeting on elective nodal irradiation in lung cancer: non-small-cell lung cancer (NSCLC). Int J Radiat Oncol Biol Phys. 2008; 72(2):335342. [PubMed: 18793953] 
3. Emami B, Mirkovic N, Scott C, et al. The impact of regional nodal radiotherapy (dose/volume) on regional progression and survival in unresectable non-small cell lung cancer: an analysis of RTOG data. Lung Cancer. 2003; 41(2):207-214. [PubMed: 12871784]

4. De Ruysscher D, Wanders S, van Haren E, et al. Selective mediastinal node irradiation based on FDG-PET scan data in patients with non-small-cell lung cancer: a prospective clinical study. Int J Radiat Oncol Biol Phys. 2005; 62(4):988-994. [PubMed: 15989999]

5. Rosenzweig KE, Sura S, Jackson A, Yorke E. Involved-field radiation therapy for inoperable nonsmall-cell lung cancer. J Clin Oncol. 2007; 25(35):5557-5561. [PubMed: 17984185]

6. Sulman EP, Komaki R, Klopp AH, Cox JD, Chang JY. Exclusion of elective nodal irradiation is associated with minimal elective nodal failure in non-small cell lung cancer. Radiat Oncol. 2009; 4:5. [PubMed: 19183471]

7. Sanuki-Fujimoto N, Sumi M, et al. Relation between elective nodal failure and irradiated volume in non-small-cell lung cancer (NSCLC) treated with radiotherapy using conventional fields and doses. Radiother Oncol. 2009; 91(3):433-437. [PubMed: 19167118]

8. Yuan S, Sun X, Li M, et al. A randomized study of involved-field irradiation versus elective nodal irradiation in combination with concurrent chemotherapy for inoperable stage III nonsmall cell lung cancer. Am J Clin Oncol. 2007; 30(3):239-244. [PubMed: 17551299]

9. Chen M, Bao Y, Ma HL, et al. Involved-field radiotherapy versus elective nodal irradiation in combination with concurrent chemotherapy for locally advanced non-small cell lung cancer: a prospective randomized study. Biomed Res Int. 2013; 2013:371819. [PubMed: 23762840]

10. Ahmed I, DeMarco M, Stevens CW, Fulp WJ, Dilling TJ. Analysis of incidental radiation dose to uninvolved mediastinal/supraclavicular lymph nodes in patients with limited-stage small cell lung cancer treated without elective nodal irradiation. Med Dosim. 2011; 36(4):440-447. [PubMed: 21397488]

11. Kepka L, Bujko K, Zolciak-Siwinska A, Garmol D. Incidental irradiation of mediastinal and hilar lymph node stations during 3D-conformal radiotherapy for non-small cell lung cancer. Acta Oncol. 2008; 47(5):954-961. [PubMed: 17899455]

12. Kong FM, Cuneo KC, Wang L, et al. Patterns of practice in radiation therapy for non-small cell lung cancer among members of the American Society for Radiation Oncology. Pract Radiat Oncol. 4(2):e133-e141. [PubMed: 24890359]

13. Bradley JD, Paulus R, Komaki R, et al. Standard-dose versus high-dose conformal radiotherapy with concurrent and consolidation carboplatin plus paclitaxel with or without cetuximab for patients with stage IIIA or IIIB non-small-cell lung cancer (RTOG 0617): a randomised, two-bytwo factorial phase 3 study. Lancet Oncol. 2015; 16(2):187-199. [PubMed: 25601342]

14. Kanzaki R, Higashiyama M, Fujiwara A, et al. Occult mediastinal lymph node metastasis in NSCLC patients diagnosed as clinical N0-1 by preoperative integrated FDG-PET/CT and CT: risk factors, pattern, and histopathological study. Lung Cancer. 2011; 71(3):333-337. [PubMed: 20619479]

15. Cerfolio RJ, Bryant AS. Survival of patients with unsuspected N2 (stage IIIA) nonsmall-cell lung cancer. Ann Thorac Surg. 2008; 86(2):362-366. discussion 366-367. [PubMed: 18640297]

16. Naruke T, Tsuchiya R, Kondo H, Nakayama H, Asamura H. Lymph node sampling in lung cancer: how should it be done? Eur J Cardiothorac Surg. 1999; 16(Suppl 1):S17-S24. [PubMed: 10536940]

17. Kotoulas CS, Foroulis CN, Kostikas K, et al. Involvement of lymphatic metastatic spread in nonsmall cell lung cancer accordingly to the primary cancer location. Lung Cancer. 2004; 44(2):183191. [PubMed: 15084383]

18. Navani N, Spiro SG, Janes SM. Mediastinal staging of NSCLC with endoscopic and endobronchial ultrasound. Nat Rev Clin Oncol. 2009; 6(5):278-286. [PubMed: 19390554]

19. Dunant A, Pignon JP, De Ruysscher D, et al. Phase III study comparing post-operative conformal radiotherapy to no postoperative radiotherapy in patients with completely resected non-small cell lung cancer and mediastinal N2 involvement. Lung Art. 2006

20. Kim JH, van Beek EJ, Murchison JT, Marin A, Mirsadraee S. The International Association for the Study of Lung Cancer lymph node map: a radiologic atlas and review. Tuberc Respir Dis (Seoul). 2015; 78(3):180-189. [PubMed: 26175770] 
21. Kong FM, Galvin J, Haken RT, Machtay M, Bradley J. Contouring atlas. RTOG Foundation Inc; 2017.

22. Marks LB, Yorke ED, Jackson A, et al. Use of normal tissue complication probability models in the clinic. Int J Radiat Oncol Biol Phys. 2010; 76(3 Suppl):S10-S19. [PubMed: 20171502]

23. Graham MV, Purdy JA, Emami B, et al. Clinical dose-volume histogram analysis for pneumonitis after 3D treatment for non-small cell lung cancer (NSCLC). Int J Radiat Oncol Biol Phys. 1999; 45(2):323-329. [PubMed: 10487552]

24. Bernard ME, Glaser SM, Gill BS, et al. Results of a single institution experience with doseescalated chemoradiation for locally advanced unresectable non-small cell lung cancer. Front Oncol. 2017; 7:1. [PubMed: 28168163]

25. Kharofa J, Gore E. Symptomatic radiation pneumonitis in elderly patients receiving thoracic irradiation. Clin Lung Cancer. 2013; 14(3):283-287. [PubMed: 23291257]

26. Qiao W-B, Zhao Y-H, Zhao Y-B, Wang R-Z. Clinical and dosimetric factors of radiation-induced esophageal injury: radiation-induced esophageal toxicity. World J Gastroenterol. 2005; 11(17): 2626-2629. [PubMed: 15849822]

27. Bradley J, Deasy JO, Bentzen S, El-Naqa I. Dosimetric correlates for acute esophagitis in patients treated with radiotherapy for lung carcinoma. Int J Radiat Oncol Biol Phys. 2004; 58(4):11061113. [PubMed: 15001251]

28. Vielva LR, Jaen MW, Alcacer JA, Cardona MC. State of the art in surgery for early stage NSCLC —does the number of resected lymph nodes matter? Transl Lung Cancer Res. 2014; 3(2):95-99. [PubMed: 25806287] 

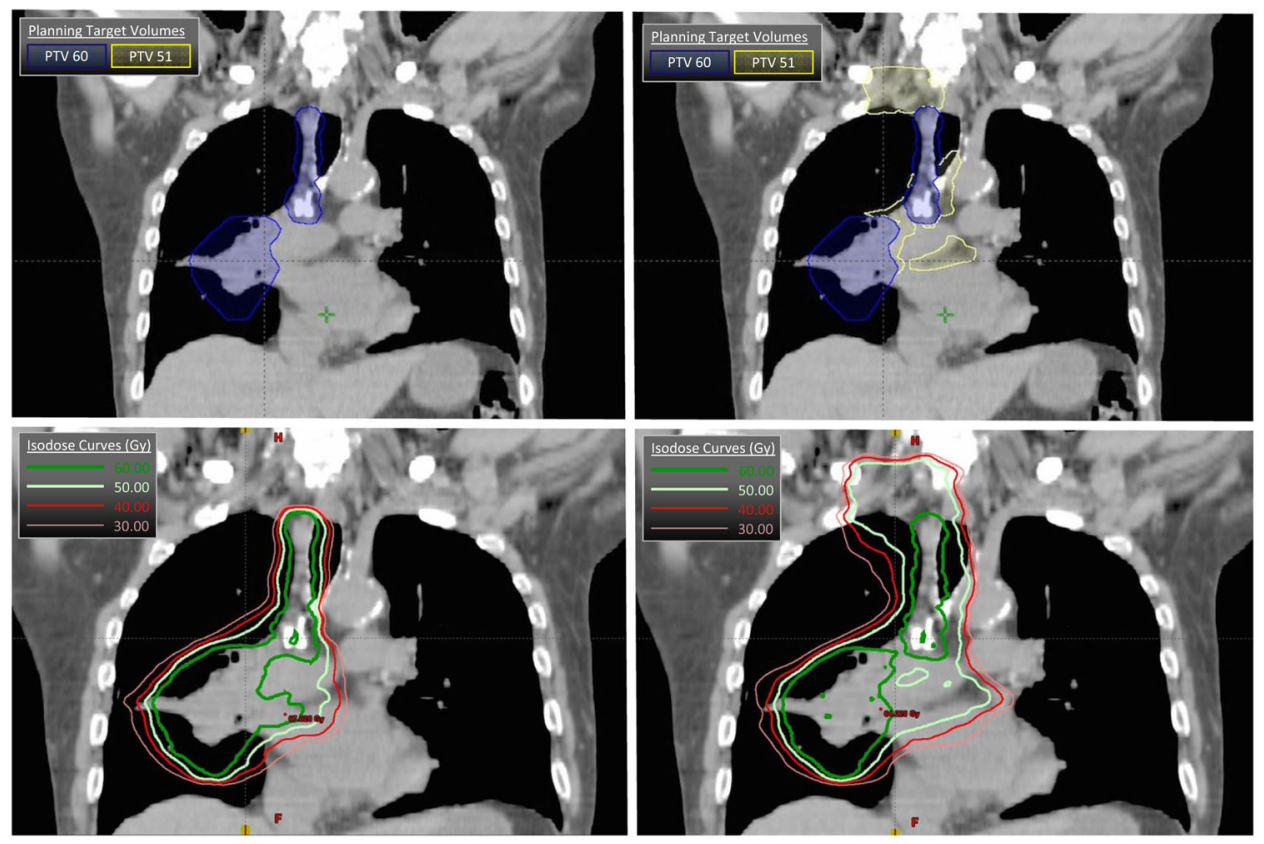

Fig. 1.

Representative planning target volumes and isodose curves for IFI (left panel) and LENI (right panel) plans for a patient with an RML primary tumor and involved nodal stations 10R, 7, 4R, and 2R 


\section{Table 1}

Thoracic nodal stations targeted for ENI based on primary tumor site and other stations involved with malignancy

\begin{tabular}{|c|c|}
\hline Primary tumor site & Elective nodal regions targeted \\
\hline \multirow[t]{3}{*}{ Right upper lobe } & All patients: $10-11 \mathrm{R}, 4 \mathrm{R}, 2 \mathrm{R}$ \\
\hline & If $2 \mathrm{R}$ involved, add $1 \mathrm{R}$ \\
\hline & If $4 \mathrm{R}$ involved, add 7 \\
\hline \multirow[t]{3}{*}{ Right middle lobe } & All patients: $10-11 \mathrm{R}, 4 \mathrm{R}, 7$ \\
\hline & If $4 R$ involved, add $2 R$ \\
\hline & If $2 \mathrm{R}$ involved, add $1 \mathrm{R}$ \\
\hline \multirow[t]{3}{*}{ Right lower lobe } & All patients: $10-11 \mathrm{R}, 4 \mathrm{R}, 7$ \\
\hline & If $4 R$ involved, add $2 R$ \\
\hline & If $2 R$ involved, add $1 R$ \\
\hline \multirow[t]{4}{*}{ Left upper lobe } & All patients: $10-11 \mathrm{~L}, 4 \mathrm{~L}, 5,6$ \\
\hline & If $4 \mathrm{~L}$ or 5 involved, add $2 \mathrm{~L}, 4 \mathrm{R}$, and 7 \\
\hline & If 6 involved, add $2 \mathrm{~L}$ \\
\hline & If $2 \mathrm{~L}$ involved, add $2 \mathrm{R}$ and $1 \mathrm{~L}$ \\
\hline \multirow[t]{3}{*}{ Left lower lobe } & All patients: $10-11 \mathrm{~L}, 4 \mathrm{~L}, 7$ \\
\hline & If $4 \mathrm{~L}$ involved, add $4 \mathrm{R}, 5,6$, and $2 \mathrm{~L}$ \\
\hline & If 7 involved, add $4 R$ \\
\hline
\end{tabular}

J Radiat Oncol. Author manuscript; available in PMC 2019 March 01. 


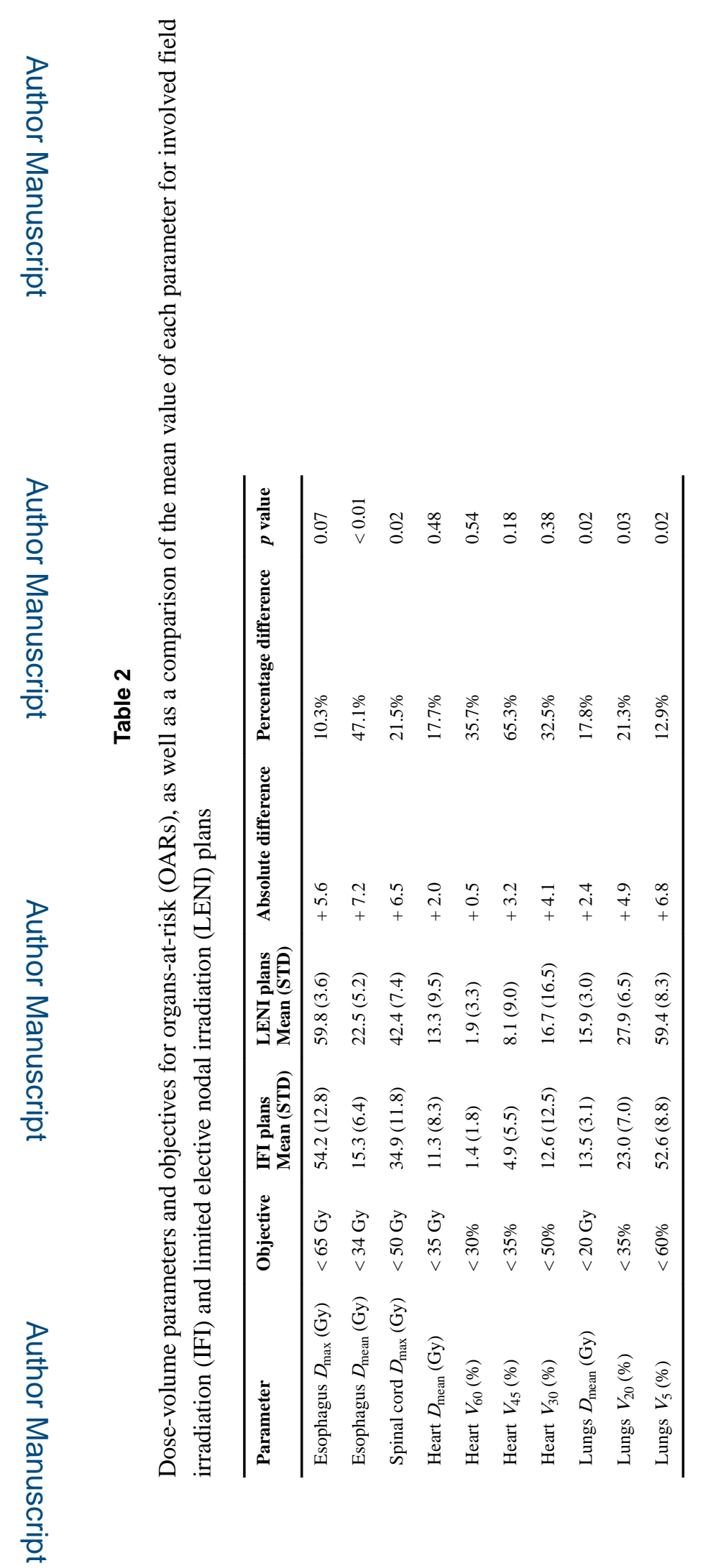

J Radiat Oncol. Author manuscript; available in PMC 2019 March 01. 


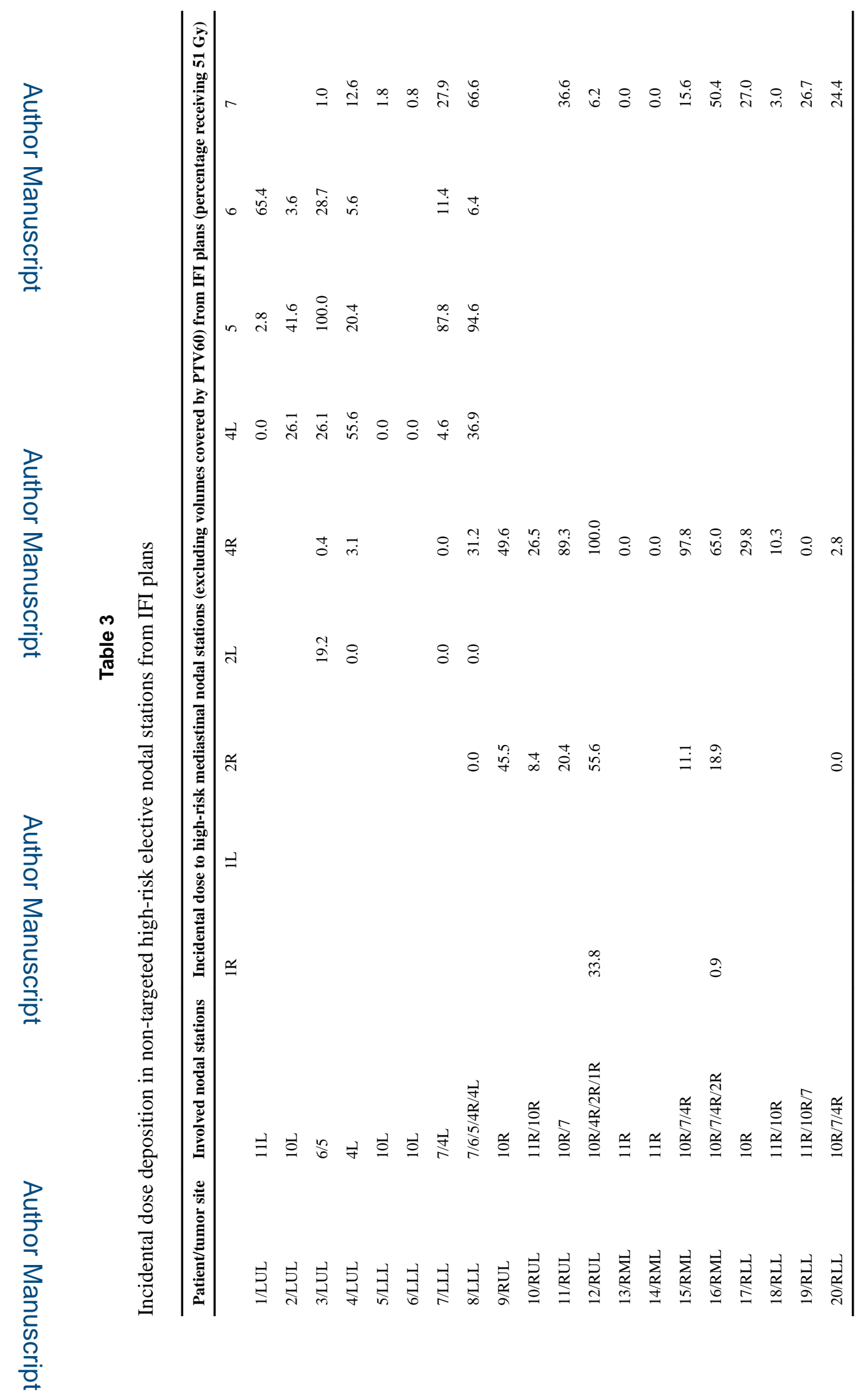

J Radiat Oncol. Author manuscript; available in PMC 2019 March 01. 